\title{
Passivity-based switching control of flexible-joint complementarity mechanical systems ${ }^{\text {और }}$
}

\author{
Constantin-Irinel Morărescu*, Bernard Brogliato \\ INRIA, BIPOP Team-project, Inovallée, 655 avenue de l'Europe, 38330, Montbonnot, France
}

In this study one considers the tracking control problem of a class of nonsmooth Lagrangian systems with flexible joints and subject to frictionless unilateral constraints. The task under consideration consists of a succession of free-motion and constrained-motion phases. Particular attention is paid to impacting and detachment phases. A passivity-based switching controller that allows one to extend the stability analysis described in our previous works to the case of systems with lumped flexibilities, is proposed. Numerical tests show the effectiveness of the controller.

\author{
Keywords: \\ Flexible joints \\ Passivity-based control \\ Nonsmooth systems \\ Lagrangian systems \\ Complementarity problem
}

\section{Introduction}

The control of mechanical systems with unilateral constraints is a challenging problem, see e.g. Galeani, Menini, Potini, and Tornambè (2008); Leine and van de Wouw (2008a,b). In this work we extend the trajectory tracking control framework developed in Brogliato, Niculescu, and Orhant (1997), Bourgeot and Brogliato (2005), Morărescu and Brogliato (2008) considering the multiconstraints flexible-joint case. The typical task representation and the stability analysis are more complex than in the rigid case because the "free-motion" controller originates from a passivitybased backstepping scheme proposed in Brogliato, Ortega, and Lozano (1995), and the study of the variations of the Lyapunov function $V(\cdot)$ arising from the backstepping method is more difficult than in the rigid case. Indeed $V(\cdot)$ cannot be forced to match the system's total energy at the impact times, and suitable upperbounds have to be computed to lead the stability analysis. The dynamics in (1) is a simplified dynamics obtained from more general Lagrangian systems using a generalized coordinate

\footnotetext{
the material in this paper was partially presented at European Control Conference, 23-26 August 2009, Budapest, Hungary. This paper was recommended for publication in revised form by Associate Editor Zongli Lin under the direction of the Editor Andrew R. Teel.

* Corresponding address: Joseph Fourier University, Laboratoire Jean Kuntzmann, LJK Site Campus - Tour IRMA, 51 rue des Mathematiques, BP 53, 38041Grenoble, France. Tel.: +33 476 514561; fax: +33 476631263 .

E-mail addresses: Constantin.Morarescu@imag.fr (C.-I. Morărescu), bernard.brogliato@inrialpes.fr (B. Brogliato).
}

transformation as in McClamroch and Wang (1988), that is supposed to hold globally in the configuration space. Notice that a nonlinear stiffness $K \mathcal{Z}(q, \theta)$ may appear due to the transformation. Details on can be found in Morărescu, Brogliato, and Haad (2008). The class of systems under consideration is expressed as:

$$
\left\{\begin{array}{l}
M(q) \ddot{q}+C(q, \dot{q}) \dot{q}+G(q)+K(q-\theta)=D^{\top} \lambda \\
J \ddot{\theta}+K(\theta-q)-K \mathcal{Z}(q, \theta)=U \\
q^{1} \geq 0,\left(q^{1}\right)^{T} \lambda=0, \lambda \geq 0 \\
\text { Collision rule }
\end{array}\right.
$$

where $q \in \mathbb{R}^{n}$ is the vector of rigid link angles, $\theta \in \mathbb{R}^{n}$ is the vector of motor shaft angles, $M(q)=M^{T}(q) \in \mathbb{R}^{n \times n}$ is a positive definite inertia matrix, $C(q, \dot{q})$ is the matrix of Coriolis and centripetal forces, $G(q)$ contains conservative forces, $\lambda \in \mathbb{R}^{m}$ is the vector of contact forces (or Lagrangian multipliers) associated with the constraints, $J \in \mathbb{R}^{n \times n}$ is the diagonal and constant matrix of the actuator inertia, $K=K^{\top}>0, K \in \mathbb{R}^{n \times n}$ represents the stiffness matrix, $U \in \mathbb{R}^{n}$ is the vector of generalized torque inputs, and $q^{1}=D q \in \mathbb{R}^{m}$ with $D=\left[\begin{array}{ll}I_{m} & 0_{m \times(n-m)}\end{array}\right]$. A constraint $i$ is said to be active if $q_{i}^{1}=0$, and inactive if $q_{i}^{1}>0$.

General notations and definitions. $\|\cdot\|$ is the Euclidean norm, $b_{p} \in \mathbb{R}^{p}$ and $b_{n-p} \in \mathbb{R}^{n-p}$ are the vectors formed with the first $p$ and the last $n-p$ components of $b \in \mathbb{R}^{n}$, respectively. $\lambda_{\min }(\cdot)$ and $\lambda_{\max }(\cdot)$ represent the smallest and the largest eigenvalues, respectively. The time-derivative of a function $f(\cdot)$ is denoted by $\dot{f}(\cdot)$. For any function $f(\cdot)$ the limit to the right at the instant $t$ will be denoted by $f\left(t^{+}\right)$and the limit to the left will be denoted by $f\left(t^{-}\right)$when they exist. A simple jump of the function $f(\cdot)$ at the moment $t=t_{\ell}$ 
is denoted $\sigma_{f}\left(t_{\ell}\right)=f\left(t_{\ell}^{+}\right)-f\left(t_{\ell}^{-}\right)$. For a real valued function $f: \mathbb{R}^{+} \mapsto \mathbb{R}$ one denotes by $S(f)$ the set of all real valued functions $g: \mathbb{R}^{+} \mapsto \mathbb{R}$ such that there exists a positive real constant $0<c<\infty$ satisfying $g(t) \leq c f(t), \forall t \geq 0$. One writes $g \in S(1) \equiv L^{\infty}$ if $f(t)=1, \forall t \geq 0.0_{n}$ is the $n$-vector with entries 0 , and $0_{n \times m}$ is the $n \times m$-zero matrix. $I_{m}$ is the $m \times m$ identity matrix A vector is considered positive if all its components are positive. A Linear Complementarity Problem (LCP) with unknown $\lambda$ is a system: $\lambda \geq 0, A \lambda+b \geq 0, \lambda^{\top}(A \lambda+b)=0$, which is compactly rewritten as $0 \leq \lambda \perp A \lambda+b \geq 0$. Such an LCP has a unique solution for all $b$ if and only if $A$ is a $P$-matrix. Positive definite matrices (not necessarily symmetric) are $P$-matrices.

Definition 1. Let $\epsilon \geq 0$ be a fixed real number. We say that a $p_{\epsilon^{-}}$ impact occurs at the instant $t$ if

$\left\|\left(q_{i}^{1}\right)_{i \in I}(t)\right\| \leq \epsilon, \quad \prod_{i \in I} q_{i}^{1}(t)=0$

where $I \subset\{1, \ldots, m\}, \operatorname{card}(I)=p$.

If $\epsilon=0$ all $p$ surfaces $\Sigma_{i}=\partial \Phi_{i}=\left\{q \in \mathbb{R}^{n} \mid q_{i}^{1}=0\right\}, i \in I$ are struck simultaneously. When $\epsilon>0$ the system collides $\partial \Phi$ in a neighborhood of the intersection $\bigcap_{i \in I} \Sigma_{i}$.

A collision (or restitution) rule is a relation between the postimpact and the pre-impact velocities. Among the various models of collision rules, Moreau's rule is an extension of Newton's law which is energetically consistent (Mabrouk, 1998) and is numerically tractable (Acary \& Brogliato, 2008). In the special coordinates of (1) this reads as $\dot{q}_{i}^{1}\left(t^{+}\right)=-e \dot{q}_{i}^{1}\left(t^{-}\right)$when $q_{1, i}(t)=0$ and $\dot{q}_{i}^{1}\left(t^{-}\right) \leq 0$, where $i \in\{1, \ldots, m\}$ and $e \in[0,1]$. Under mild conditions on the data, the solutions are such that positions $q(\cdot)$ and $\theta(\cdot)$ are absolutely continuous functions of time, whereas the generalized velocity is right continuous of local bounded variation (Dzonou \& Monteiro Marques, 2007). The continuity of $\dot{\theta}(\cdot)$ holds (Brogliato, 1999) and will be used in the stability analysis developed in Section 5 .

\section{Basic concepts}

Since the system's dynamics does not change when the number of active constraints decreases one gets the following typical task representation:

$\mathbb{R}_{+}=\Omega_{0}^{\emptyset} \cup\left[\bigcup_{k \geq 1}\left(I_{k} \cup\left(\bigcup_{i=1}^{m_{k}} \Omega_{k}^{B_{k, i}}\right)\right)\right]$

$B_{k, m_{k}} \subset B_{k+1,1}, B_{k, m_{k}} \subset B_{k, m_{k}-1} \subset \cdots \subset B_{k, 1}$

where the superscript $B_{k}$ represents the set of active constraints $\left(B_{k}=\left\{i \in\{1, \ldots, m\} \mid F_{i}(X)=0\right\}\right)$ during the corresponding motion phase $\Omega_{k}$, and $I_{k}^{B_{k}}$ denotes the transient between two $\Omega_{k}$ phases ${ }^{1}$ when the number of active constraints increases. We note that $B_{k}=\varnothing$ corresponds to free-motion. When the number of active constraints decreases there is no impact, thus no other transition phases are needed. Throughout the paper, the sequence $I_{k} \cup\left(\bigcup_{i=1}^{m_{k}} \Omega_{k}^{B_{k, i}}\right)$ will be referred to as the cycle $k$ of the system's evolution.

\subsection{System properties}

For kinematic chains with prismatic or revolute joints the following properties hold.

\footnotetext{
1 To simplify the presentation the phases of motion and the time intervals that correspond to the phases are denoted identically.
}

Property 1. The matrix $\left[\frac{\mathrm{d}}{\mathrm{d} t} M(q)\right]-2 C(q, \dot{q})$ is skew-symmetric and $\dot{M}(q) \triangleq \frac{\mathrm{d}}{\mathrm{d} t} M(q)=C(q, \dot{q})+C^{\top}(q, \dot{q})$. Furthermore the matrix $C(q, \dot{q})$ is a smooth function of $q$ and $\dot{q}$ with the well-known properties $\|C(q, \dot{q})\| \in S(\|\dot{q}\|)$ and $C(q, y) z=C(q, z) y, \quad \forall q, y, z \in \mathbb{R}^{n}$.

Property 2. The conservative forces vector $G(q)$ is such that $\left\|\frac{\partial G(q)}{\partial q}\right\| \in S(1)$ which implies by the mean value theorem $\| G\left(q_{1}\right)$ $G\left(q_{2}\right) \| \in S\left(\left\|q_{1}-q_{2}\right\|\right), \quad \forall q_{1}, q_{2} \in \mathbb{R}^{n}$.

Property 3. The matrix $C(q, \dot{q})$ is such that $\left\|\frac{\partial C(q, \dot{q})}{\partial q}\right\| \in S(\|\dot{q}\|)$ and $\left\|\frac{\partial C(q, \dot{q})}{\partial \dot{q}}\right\| \in S(1)$.

\subsection{Design of the exogenous trajectories}

Throughout the paper, the following trajectories will play a role in the closed-loop dynamics:

- $q^{n c}(\cdot)$ denotes the desired trajectory that the system should track if there were no constraints. We suppose that $q^{1, n c}(t)<$ 0 for some $t$, otherwise the problem reduces to the tracking control of a system with no constraints.

- $q_{d}^{*}(\cdot)$ denotes the signal entering the control input and playing the role of the desired trajectory during some parts of the motion.

- $q_{d}(\cdot)$ represents the signal entering the Lyapunov function $V(\cdot)$. This signal is set on the boundary $\partial \Phi$ after the first impact of each cycle.

We always consider $I_{k}=\left[\tau_{0}^{k}, t_{f}^{k}\right]$ where $\tau_{0}^{k}$ is chosen by the designer as the start of the transition phase $I_{k}$ and $t_{f}^{k}$ is the end of this phase. During the transition phases the system must be stabilized on the intersection of some surfaces $\Sigma_{i}$. This will be done by mimicking the behavior of a ball falling on the ground under gravity. Therefore all the components except the ones that are normal to the constraints belonging to $B_{k}$ will be frozen. Moreover for robustness reasons one avoids a tangential approach and imposes some impacts defining an exogenous signal $q_{d}^{*}$ that violates the constraints.

In order to simplify the presentation we introduce the following notations (where all superscripts $(\cdot)^{k}$ will refer to the cycle $k$ of the system motion):

- $t_{0}^{k}$ is the first impact during the cycle $k$,

- $t_{\infty}^{k}$ is the accumulation point of the sequence $\left\{t_{\ell}^{k}\right\}_{\ell \geq 0}$ of the impact instants during the cycle $k\left(t_{f}^{k} \geq t_{\infty}^{k}\right)$,

- $\tau_{1}^{k}$ will be explicitly defined later and represents the instant when the exogenous signal $q_{d}^{*}$ reaches a given value chosen by the designer in order to impose a closed-loop dynamics with impacts during the transition phases,

- $t_{d}^{k, i}$ is the desired detachment instant at the end of $\Omega_{k}^{J_{k, i}}$.

It is noteworthy that $t_{0}^{k}, t_{\infty}^{k}$ are state-dependent whereas $\tau_{1}^{k}, \tau_{0}^{k}$ and $t_{d}^{k, i}$ are exogenous and imposed by the designer.

\subsection{Stability analysis criteria}

Let us define $\Omega$ as the complement in $\mathbb{R}_{+}$of $I=\bigcup_{k>0} I_{k}$ and assume that the Lebesgue measure of $\Omega$, denoted $\lambda[\Omega]$, equals infinity. Let $x(\cdot)$ be the state of the closed-loop system in (1) with some feedback controller $U(q, \dot{q}, \theta, \dot{\theta}, t)$.

Definition 2. The closed loop system is called practically weakly stable if there exists $0<R<+\infty$ and $t^{*}<+\infty$ such that $\|x(t)\|<R$ for all $t>t^{*}, t \in \Omega$. 
Consider $V(\cdot)$ such that there exist strictly increasing functions $\alpha(\cdot)$ and $\beta(\cdot)$ satisfying the conditions: $\alpha(0)=0, \beta(0)=0$ and $\alpha(\|x\|) \leq V(x, t) \leq \beta(\|x\|)$. The following result was proved in Morărescu et al. (2008):

Proposition 1. Assume that the task admits the representation (2) and that

(a) $t_{f}^{k}<t_{d}^{k, 1}, \quad \forall k \in \mathbb{N}$,

(b) outside the impact accumulation phases $\left[t_{0}^{k}, t_{\infty}^{k}\right]$ one has $\dot{V}(x(t), t) \leq-\gamma V(x(t), t)$ for some constant $\gamma>0$,

(c) the system is initialized on $\Omega_{0}$ such that $V\left(\tau_{0}^{0}\right) \leq 1$,

(d) $V\left(t_{\infty}^{k}\right) \leq \rho^{*} V\left(\tau_{0}^{k}\right)+\xi$ where $\rho^{*}, \xi \in \mathbb{R}_{+}$.

Then $V\left(\tau_{0}^{k}\right) \leq \delta(\gamma, \xi), \forall k \geq 1$ where $\delta(\gamma, \xi)$ is a function that can be made arbitrarily small by increasing either the value of $\gamma$ or the length of the time interval $\left[t_{\infty}, t_{f}\right]$. Thus, the system is practically weakly stable with $R=\alpha^{-1}(\delta(\gamma, \xi))$.

It is worth to point out that condition (d) means that the impacts may be considered as a kind of disturbance that can be suitably upper bounded. This is certainly the most crucial point in Proposition 1.

\section{Controller design}

Let us introduce the notations: $\left(\begin{array}{l}\tilde{q} \\ \tilde{\theta}\end{array}\right)=\left(\begin{array}{l}q-q_{d} \\ \theta-\theta_{d}\end{array}\right), s=\left(\begin{array}{l}s_{1} \\ s_{2}\end{array}\right)=$ $\left(\begin{array}{c}\dot{\tilde{q}}+\gamma_{2} \tilde{q} \\ \tilde{\theta}+\gamma_{2} \tilde{\theta}\end{array}\right), \dot{q}_{r}=\dot{q}_{d}-\gamma_{2} \tilde{q}, \bar{q}=q-q_{d}^{*}$ and $\bar{s}_{1}=\dot{\bar{q}}+\gamma_{2} \bar{q}$, where $\gamma_{2}>0$ is a scalar gain. The tracking problem is solved using a generalization of the controller proposed in Brogliato et al. (1995, Equ. (28)) and the closed-loop stability analysis of the system is based on Proposition 1. The controller is defined by

$$
\left\{\begin{array}{l}
U=J \ddot{\theta}_{r}+K\left(\theta_{d}-q_{d}\right)-\gamma_{1} s_{2}-K \mathcal{Z}(q, \theta) \\
\theta_{d}=q_{d}+K^{-1} U_{r}
\end{array}\right.
$$

where $U_{r}$ is given by:

$U_{r}=\left\{\begin{array}{l}U_{c}^{\emptyset} \triangleq U_{n c}=M(q) \ddot{q}_{r}+C(q, \dot{q}) \dot{q}_{r}+G(q)-\gamma_{1} s_{1} \\ \quad \text { for } t \in \Omega_{2 k}^{\emptyset} \\ U_{c}^{B_{k}}=U_{n c}-P_{d}+K_{f}\left(P_{q}-P_{d}\right) \quad \text { for } t \in \Omega_{k}^{B_{k}} \\ U_{c}^{B_{k}} \text { for } t \in I_{k} \text { before the first impact } \\ U_{t}^{B_{k}}=M(q) \ddot{q}_{r}+C(q, \dot{q}) \dot{q}_{r}+G(q)-\gamma_{1} \bar{s}_{1} \\ \quad \text { for } t \in I_{k} \text { after the first impact }\end{array}\right.$

where $\gamma_{1}>0$ is a scalar gain, $K_{f}>0, P_{q}=D^{T} \lambda$ and $P_{d}=D^{T} \lambda_{d}$ is the desired contact force during the persistently constrained motion. It is clear that during $\Omega_{k}^{B_{k}}$ not all the constraints are active and, therefore, some components of $\lambda$ and $\lambda_{d}$ are zero. Notice that on impacting phases no force feedback is applied. Also $U$ is a function of $q, \theta, \dot{q}, \dot{\theta}$ only (no acceleration feedback). The rationale behind the change of structure of $U_{r}$ after the first impact, is that it facilitates the calculation of some upper-bounds which are necessary to recast the closed-loop stability analysis into Proposition 1 (see Section 5 and the Appendix). In order to prove the stability of the closed-loop system (1), (3) and (4) we will use the following positive definite function:

$$
\begin{aligned}
V(t, s, \tilde{q}, \tilde{\theta})= & \frac{1}{2} s_{1}^{T} M(q) s_{1}+\frac{1}{2} s_{2}^{T} J s_{2}+\gamma_{1} \gamma_{2} \tilde{q}^{T} \tilde{q} \\
& +\gamma_{1} \gamma_{2} \tilde{\theta}^{T} \tilde{\theta}+\frac{1}{2}(\tilde{q}-\tilde{\theta})^{T} K(\tilde{q}-\tilde{\theta}) .
\end{aligned}
$$

\section{Tracking control strategy}

The design of $q_{d}^{*}(\cdot)$ and $q_{d}(\cdot)$ during the phases $I_{k}$, the design of the desired contact force during constraint phases and the strategy for take-off at the end of constraint phases are similar to those proposed in the rigid case (see Morărescu \& Brogliato, 2008). Therefore, we briefly present the results and some discussions without giving the proof details.

During the impacting transition phases the system must be stabilized on $\partial \Phi$. Obviously, this does not mean that all the constraints have to be activated (i.e. $q_{i}^{1}(t)=0, \forall i=1, \ldots, m$ ). Let us consider that only the first $p$ constraints (eventually reordering the coordinates) define the border of $\Phi$ where the system must be stabilized. The signal $q_{d}^{*}(\cdot)$ will be then defined as follows:

- choosing $v>0$ and denoting $t^{\prime}=\frac{t-\tau_{0}^{k}}{\tau_{1}^{k}-\tau_{0}^{k}}$, the components $\left(q_{d}^{i}\right)^{*}, i=1, \ldots, p$ of $\left(q_{d}^{*}\right)_{p}$ are defined as:

$$
\left(q_{d}^{i}\right)^{*}(t)=\left\{\begin{array}{l}
a_{3}\left(t^{\prime}\right)^{3}+a_{2}\left(t^{\prime}\right)^{2}+a_{0}, \quad t \in\left[\tau_{0}^{k}, \min \left\{\tau_{1}^{k} ; t_{0}^{k}\right\}\right] \\
-v V^{1 / 3}\left(\tau_{0}^{k}\right), \quad t \in\left(\min \left\{\tau_{1}^{k} ; t_{0}^{k}\right\}, t_{f}^{k}\right]
\end{array}\right.
$$

where $V(\cdot)$ is defined in (5) and $\tau_{1}^{k}$ is chosen by the designer such that the limit condition $\left(q_{d}^{i}\right)^{*}\left(\tau_{1}^{k}\right)=-v V^{1 / 3}\left(\tau_{0}^{k}\right),\left(\dot{q}_{d}^{i}\right)^{*}$ $\left(\tau_{1}^{k}\right)=0$ holds, which allows the computation of the previous coefficients as:

$$
\begin{aligned}
& a_{3}=2\left[\left(q^{i}\right)^{n c}\left(\tau_{0}^{k}\right)+v V^{1 / 2}\left(\tau_{0}^{k}\right)\right] \\
& a_{2}=-3\left[\left(q^{i}\right)^{n c}\left(\tau_{0}^{k}\right)+v V^{1 / 2}\left(\tau_{0}^{k}\right)\right] \\
& a_{0}=\left(q^{i}\right)^{n c}\left(\tau_{0}^{k}\right)
\end{aligned}
$$

- all the other components of $q_{d}^{*}(\cdot)$ are frozen on $I_{k}$, i.e.:

$$
\left(q_{d}^{*}\right)_{n-p}(t)=q_{n-p}^{n c}\left(\tau_{0}^{k}\right), \quad t \in\left(\tau_{0}^{k}, t_{f}^{k}\right]
$$

In order to limit the deformation of the desired trajectory $q_{d}^{*}(\cdot)$ w.r.t. $q^{n c}(\cdot)$ during the $I_{k}$ phases, we impose in the sequel

$\left\|q_{p}^{n c}\left(\tau_{0}^{k}\right)\right\| \leq v_{1}$

where $v_{1}>0$ is chosen by the designer. A smaller $v_{1}$ leads to a smaller deformation of the desired trajectory and to a smaller deformation of the real trajectory. Nevertheless, due to the tracking error, $v_{1}$ cannot be chosen to be zero. We also note that (9) is a practical way to choose $\tau_{0}^{k}$.

During the transition phases $I_{k}$ we define $\left(q_{d}\right)_{n-p}(t)=$ $\left(q_{d}^{*}\right)_{n-p}(t)$. Assuming a finite accumulation period, the impact process can be considered in some way equivalent to a plastic impact. Therefore, $\left(q_{d}\right)_{p}(\cdot)$ and $\left(\dot{q}_{d}\right)_{p}(\cdot)$ are set to zero on the right of $t_{0}^{k}$. It is worth recalling that the first impact time $t_{0}^{k}$ of each cycle $k$, is unknown.

The desired contact force $P_{d}=D^{\top} \lambda_{d}$ must be designed such that it is large enough to assure the constraint motion on the $\Omega_{k}^{B_{k}}$ phases. Some contact force components have also to be decreased at the end of the $\Omega_{k}^{B_{k}}$-phases in order to allow the detachment. Therefore we need a lower bound of the desired force which assures both contact (without any undesired detachment which can generate other impacts) during the $\Omega_{k}^{B_{k}}$ phases and a smooth detachment at the end of $\Omega_{k}^{B_{k}}$. Dropping the time argument, the dynamics of the system on $\Omega_{k}^{B_{k}}$ can be written as

$$
\left\{\begin{array}{l}
M(q) \ddot{q}+F=\left(1+K_{f}\right) D_{p}^{\top}\left(\lambda-\lambda_{d}\right) \\
J \dot{s}_{2}+\gamma_{1} s_{2}+K(\tilde{\theta}-\tilde{q})=0 \\
0 \leq q_{p} \perp \lambda_{p} \geq 0
\end{array}\right.
$$


where $F=F(q, \dot{q}, \tilde{q}, \dot{\tilde{q}}, \tilde{\theta})=-M(q) \ddot{q}_{r}+C(q, \dot{q}) s_{1}+\gamma_{1} s_{1}+K(\tilde{q}-\tilde{\theta})$ and $D_{p}=\left[I_{p} \vdots O_{p \times(n-p)}\right] \in \mathbb{R}^{p \times n}$. On $\Omega_{k}^{B_{k}}$ the system has to be permanently constrained which is equivalent to $q_{p}(\cdot)=0$ and $\dot{q}_{p}(\cdot)=0$. In order to assure these conditions it is sufficient to have $\lambda_{p}>0$.

We denote

$M^{-1}(q)=\left(\begin{array}{ll}{\left[M^{-1}(q)\right]_{p, p}} & {\left[M^{-1}(q)\right]_{p, n-p}} \\ {\left[M^{-1}(q)\right]_{n-p, p}} & {\left[M^{-1}(q)\right]_{n-p, n-p}}\end{array}\right)$

and

$C(q, \dot{q})=\left(\begin{array}{ll}C(q, \dot{q})_{p, p} & C(q, \dot{q})_{p, n-p} \\ C(q, \dot{q})_{n-p, p} & C(q, \dot{q})_{n-p, n-p}\end{array}\right)$

where the meaning of each component is obvious. Let us also denote by $K_{p}$ the matrix made of the first $p$ rows and $p$ columns of $K$.

Proposition 2. On $\Omega_{k}^{B_{k}}$ the constraint motion of the closed-loop system (10), (3) and (4) is assured if the desired contact force is defined by

$$
\begin{aligned}
\left(\lambda_{d}\right)_{p} \triangleq & v_{p}+\frac{K_{p} \tilde{\theta}_{p}}{1+K_{f}}-\frac{\bar{M}_{p, p}(q)}{1+K_{f}}\left(\left[M^{-1}(q)\right]_{p, p} C_{p, n-p}(q, \dot{q})\right. \\
& \left.+\left[M^{-1}(q)\right]_{p, n-p}\left(C_{n-p, n-p}(q, \dot{q})+\gamma_{1} I_{n-p}\right)\right)\left(s_{1}\right)_{n-p}
\end{aligned}
$$

where $\bar{M}_{p, p}(q)=\left(\left[M^{-1}(q)\right]_{p, p}\right)^{-1}=\left(D_{p} M^{-1}(q) D_{p}^{T}\right)^{-1}$ and $v_{p} \in$ $\mathbb{R}^{p}, v_{p}>0$.

Let us now consider that the first $h$ constraints $(h<p)$ have to be deactivated. Thus, the detachment takes place at $t_{d}^{k}$ if $\ddot{q}_{h}\left(t_{d}^{k+}\right)>$ 0 which requires $\lambda_{h}\left(t_{d}^{k-}\right)=0$. The last $p-h$ constraints remain active which means $\lambda_{p-h}\left(t_{d}^{k-}\right)>0$. To simplify the notation we drop the time argument in many equations of this section. We decompose the LCP matrix as:

$\left(1+K_{f}\right) D_{p} M^{-1}(q) D_{p}^{T}=\left(\begin{array}{ll}A_{1}(q) & A_{2}(q) \\ A_{2}(q)^{T} & A_{3}(q)\end{array}\right)$

with $A_{1} \in \mathbb{R}^{h \times h}, A_{2} \in \mathbb{R}^{h \times(p-h)}$ and $A_{3} \in \mathbb{R}^{(p-h) \times(p-h)}$.

Proposition 3. The closed-loop system (10), (3) and (4) is permanently constrained on $\left[t_{f}^{k}, t_{d}^{k}\right)$ and a smooth detachment is guaranteed on $\left[t_{d}^{k}, t_{d}^{k}+\epsilon\right)(\epsilon$ is a small positive real number chosen by the designer) if

$$
\begin{aligned}
& \left(\begin{array}{c}
\left(\lambda_{d}\right)_{h}\left(t_{d}^{k}\right) \\
\left(\lambda_{d}\right)_{p-h}\left(t_{d}^{k}\right)
\end{array}\right) \\
& =\left(\begin{array}{c}
\left(A_{1}-A_{2} A_{3}^{-1} A_{2}^{T}\right)^{-1}\left(b_{h}-A_{2} A_{3}^{-1} b_{p-h}\right)-C_{1}\left(t-t_{d}^{k}\right) \\
C_{2}+A_{3}^{-1}\left(b_{p-h}-A_{2}^{T}\left(\lambda_{d}\right)_{h}\right)
\end{array}\right)
\end{aligned}
$$

where

$b_{p} \triangleq b\left(q, \dot{q}, U_{c}^{\emptyset}\right) \triangleq-D_{p} M^{-1}(q) F \geq 0$

and $C_{1} \in \mathbb{R}^{h}, C_{2} \in \mathbb{R}^{p-h}$ such that $C_{1} \geq 0, C_{2}>0$.

Furthermore on $\left[t_{d}^{k}, t_{d}^{k}+\epsilon\right)$

$q_{d}^{*}(t)=q_{d}(t)=\left(\begin{array}{c}q_{h}^{*}(t) \\ q_{n-h}^{n c}(t)\end{array}\right)$,

where $q_{h}^{*}(\cdot)$ is a twice-differentiable function such that

$q_{h}^{*}\left(t_{d}^{k}\right)=0, \quad q_{h}^{*}\left(t_{d}^{k}+\epsilon\right)=q_{h}^{n c}\left(t_{d}^{k}+\epsilon\right)$,

$\dot{q}_{h}^{*}\left(t_{d}^{k}\right)=0, \quad \dot{q}_{h}^{*}\left(t_{d}^{k}+\epsilon\right)=\dot{q}_{h}^{n c}\left(t_{d}^{k}+\epsilon\right)$

and $\ddot{q}_{h}^{*}\left(t_{d}^{k+}\right)=a>\max \left(0,-A_{1}(q)\left(\lambda_{d}\right)_{h}\left(t_{d}^{k-}\right)\right)$.

\section{Closed-loop stability analysis}

For convenience $V(t, s(t), \tilde{q}, \tilde{\theta}(t))$ is written as $V(t)$.

Lemma 1. Consider the closed-loop system (1), (3) and (4) with $\left(q_{d}^{*}\right)_{p}(\cdot)$ defined on the interval $\left[\tau_{0}^{k}, t_{0}^{k}\right]$ as in (6)-(8). Let us also suppose that condition (b) of Proposition 1 is satisfied. The following inequalities hold:

$$
\begin{aligned}
& \left\|\tilde{q}\left(t_{0}^{k-}\right)\right\| \leq \sqrt{\frac{V\left(\tau_{0}^{k}\right)}{\gamma_{1} \gamma_{2}}}, \quad\left\|s_{1}\left(t_{0}^{k-}\right)\right\| \leq \sqrt{\frac{2 V\left(\tau_{0}^{k}\right)}{\lambda_{\min }(M(q))}}, \\
& \left\|\tilde{\theta}\left(t_{0}^{k-}\right)\right\| \leq \sqrt{\frac{V\left(\tau_{0}^{k}\right)}{\gamma_{1} \gamma_{2}}}, \quad\left\|s_{2}\left(t_{0}^{k-}\right)\right\| \leq \sqrt{\frac{2 V\left(\tau_{0}^{k}\right)}{\lambda_{\min }(J)}}, \\
& \left\|\dot{\tilde{q}}\left(t_{0}^{k-}\right)\right\| \leq\left(\sqrt{\frac{2}{\lambda_{\min }(M(q))}}+\sqrt{\frac{\gamma_{2}}{\gamma_{1}}}\right) V^{1 / 2}\left(\tau_{0}^{k}\right) \\
& \left\|\dot{\tilde{\theta}}\left(t_{0}^{k-}\right)\right\| \leq\left(\sqrt{\frac{2}{\lambda_{\min }(J)}}+\sqrt{\frac{\gamma_{2}}{\gamma_{1}}}\right) V^{1 / 2}\left(\tau_{0}^{k}\right) .
\end{aligned}
$$

Furthermore, if $t_{0}^{k} \leq \tau_{1}^{k}$ one has

$$
\begin{aligned}
& \left\|\left(q_{d}\right)_{p}\left(t_{0}^{k-}\right)\right\| \leq \epsilon+\sqrt{\frac{V\left(\tau_{0}^{k}\right)}{\gamma_{1} \gamma_{2}}}, \\
& \left\|\left(\dot{q}_{d}\right)_{p}\left(t_{0}^{k-}\right)\right\| \leq \bar{k}+k^{*} V^{1 / 6}\left(\tau_{0}^{k}\right) \\
& \left\|\left(\ddot{q}_{d}\right)_{p}\left(t_{0}^{k-}\right)\right\| \leq 6 \sqrt{2}\left(\left\|q_{p}^{n c}\left(\tau_{0}^{k}\right)\right\|+\sqrt{p} v V^{1 / 2}\left(\tau_{0}^{k}\right)\right) \\
& \left\|\left(q_{d}^{(3)}\right)_{p}\left(t_{0}^{k-}\right)\right\| \leq 6 \sqrt{2}\left(\left\|q_{p}^{n c}\left(\tau_{0}^{k}\right)\right\|+\sqrt{p} v V^{1 / 2}\left(\tau_{0}^{k}\right)\right)
\end{aligned}
$$

where $\epsilon$ is the real constant fixed in Definition 1 ,

$$
\begin{gathered}
\bar{k}=\frac{6 \sqrt{p v_{1} \epsilon}}{\tau_{1}^{k}-\tau_{0}^{k}} \text { and } \\
k^{*}=\frac{6 \sqrt{p}}{\tau_{1}^{k}-\tau_{0}^{k}} \sqrt{\left(\frac{1}{\sqrt{\gamma_{1} \gamma_{2}}}+v\right)\left(v+v_{1}\right)+\epsilon v .}
\end{gathered}
$$

Proof. The proof closely follows the ideas presented in Morărescu and Brogliato (2008) and is omitted. The details can be found in Morărescu et al. (2008).

Lemma 2. The controller $U$ in (3) and (4) guarantees that $\left\|\sigma_{\tilde{\theta}}(\cdot)\right\|$, $\left\|\sigma_{\dot{\tilde{\theta}}}(\cdot)\right\| \in S(1) \equiv L^{\infty}$.

Proof. See Appendix A.

Theorem 1. Let $e=0$ and $q_{d}^{*}(\cdot)$ defined as in (6)-(8). The closedloop system (1), (3) and (4) initialized on $\Omega_{0}$ such that $V\left(\tau_{0}^{0}\right) \leq 1$, satisfies the requirements of Proposition 1 and is therefore practically weakly stable with the closed-loop state $x(\cdot)=[\tilde{q}, \tilde{\theta}(\cdot), s(\cdot)]$ and $R=\sqrt{\mathrm{e}^{-\gamma\left(t_{f}^{k}-t_{\infty}^{k}\right)}\left(\rho^{*}+\xi\right) / \bar{\rho}}$ where $\rho^{*}, \bar{\rho}$ and $\xi$ are defined in the proof.

Proof. See Appendix B.

\section{Illustrative example}

We simulate the behavior of a planar two-link flexible-joint manipulator in presence of two constraints. The admissible domain is $\Phi=\{(x, y) \mid y \geq 0,0.7-x \geq 0\}$. The unconstrained desired trajectory is given by the circle $\left\{(x, y) \mid(x-0.7)^{2}+y^{2}=0.5\right\}$ and violates both constraints. In other words, the two-link planar 

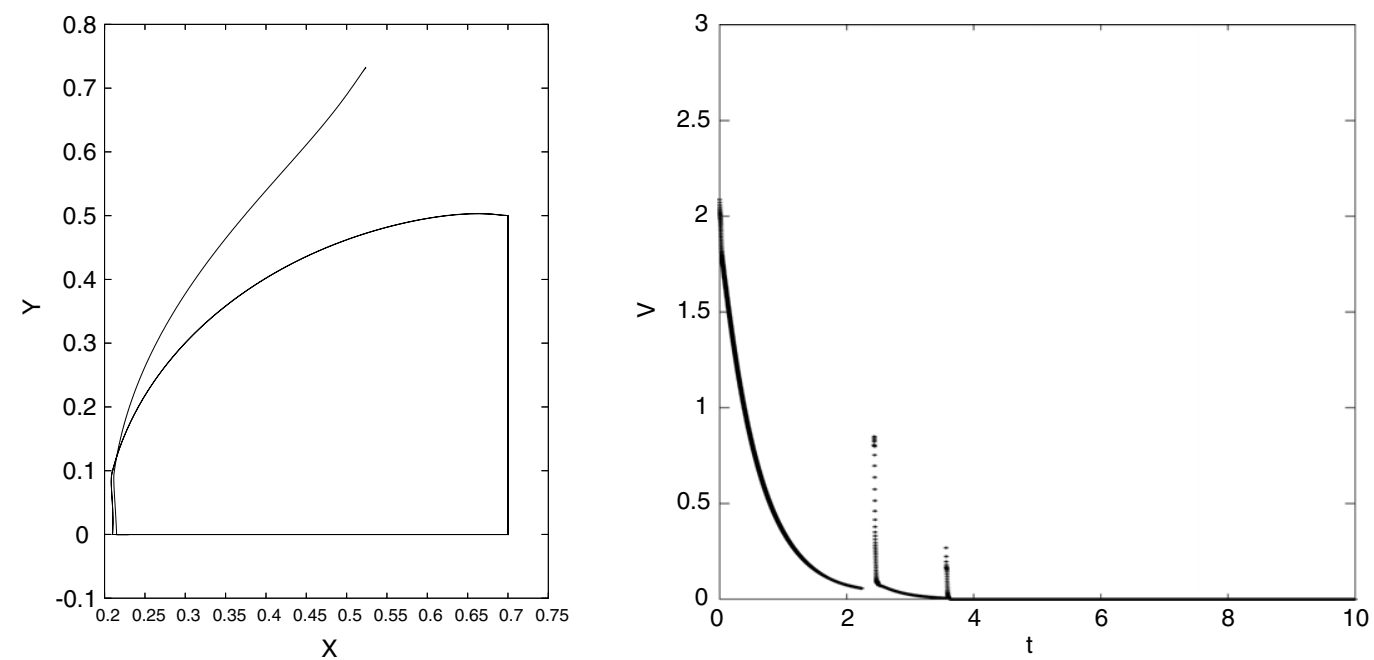

Fig. 1. Left: The trajectory of the system during 6 rounds; Right: The variation of the almost non-increasing Lyapunov function during the first round.

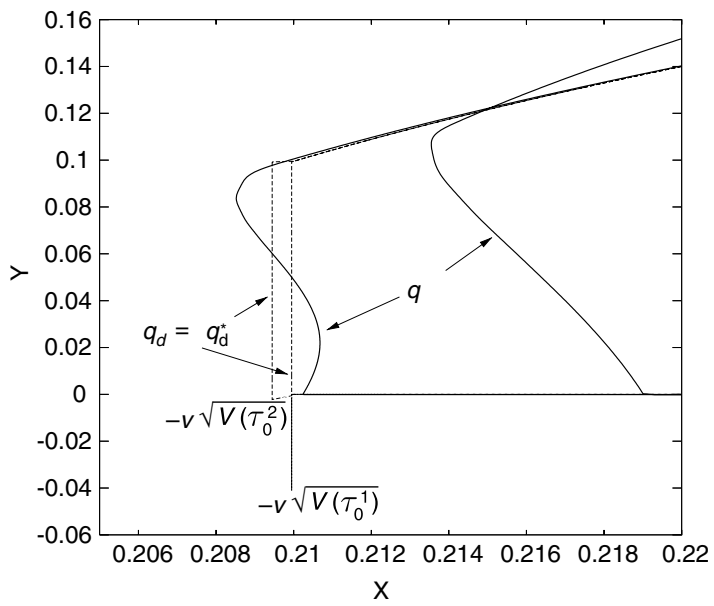

Fig. 2. Zoom on the transition phases $I_{1}$ and $I_{3}$.

manipulator must track a quarter-circle; stabilize on and then follow the line $\Sigma_{1}=\{(x, y) \mid y=0\}$; stabilize on the intersection of $\Sigma_{1}$ and $\Sigma_{2}=\{(x, y) \mid x=0.7\}$; detach from $\Sigma_{1}$ and follow $\Sigma_{2}$ until the unconstrained circle re-enters $\Phi$ and finally take-off from $\Sigma_{2}$ in order to repeat the previous steps. Therefore, we have: $\mathbb{R}^{+}=$ $\Omega_{0}^{\emptyset} \cup I_{1} \cup \Omega_{1}^{J_{1,1}} \cup I_{2} \cup \Omega_{2}^{J_{2,1}} \cup \Omega_{2}^{J_{2,2}} \cup \Omega_{2}^{J_{2,3}} \cup I_{3} \cup \Omega_{2}^{J_{3,1}} \cup I_{4} \cup \cdots$ with $J_{1,1}=$ $\{1\}, J_{2,1}=\{1,2\}, J_{2,2}=\{2\}, J_{2,3}=\emptyset$, etc. We note that during $I_{2 k+1}$ the system is stabilized on $\Sigma_{1}$ (1-impacts) while during $I_{2 k}$ the system is stabilized on $\Sigma_{1} \cap \Sigma_{2}\left(2_{\epsilon_{k}}\right.$-impacts). The numerical values used for the dynamical model are $l_{1}=l_{2}=0.5 \mathrm{~m}, m_{1}=$ $m_{2}=1 \mathrm{~kg}, I_{1}=I_{2}=0.5 \mathrm{~kg} \mathrm{~m}^{2}, J_{1}=J_{2}=0.1 \mathrm{~kg} \mathrm{~m}^{2}$ and the impacts are imposed by $v=10$ in (6) and (7). The stiffness matrix is defined by $K=\operatorname{diag}(2000 \mathrm{~N} \mathrm{~m}, 2000 \mathrm{~N} \mathrm{~m})$. Let us say that the quarter-circle is completely tracked in one round. We set the period of each round to $10 \mathrm{~s}$ and we simulate the dynamics during 6 rounds using the Moreau's time-stepping algorithm of the Siconos software platform (Acary \& Brogliato, 2008). We set the controller gains $\gamma_{1}=10, \gamma_{2}=1$ and we choose $v_{1}=0.1$ (like this we implicitly set $\tau_{0}^{k}$ see (9)) in order to better point out the deformation of $q_{d}(\cdot)$ on the transition phases (Fig. 1 (left)). In Fig. 2 we have shifted the desired trajectory backwards on $I_{3}$ to highlight that the Lyapunov function at the instant $\tau_{0}^{k}$ is smaller when $k$ increases. The behavior of the system during one round is emphasized in Fig. 1 (right).

\section{Conclusions}

In this paper we have proposed a solution for the control of nonsmooth Lagrangian systems with flexible joints. All the ingredients entering the dynamics (desired trajectories, desired contact forces, exogenous instants playing a role in the definition of the control law) are explicitly defined. Numerical simulations are done with the software platform in order to illustrate the results.

\section{Acknowledgement}

The first author's work was supported by the European project SICONOS FP5 IST-2001-37172.

\section{Appendix A. Proof of Lemma 2}

Since $\theta(\cdot), \dot{\theta}(\cdot)$ are continuous on $\mathbb{R}_{+}$and $\theta_{d}(\cdot), \dot{\theta}_{d}(\cdot)$ are continuous on $\mathbb{R}_{+} \backslash\left\{t_{0}^{k} \mid k \in \mathbb{Z}\right\}$ one deduces that $\sigma_{\tilde{\theta}}(t)=0=\sigma_{\dot{\tilde{\theta}}}(t)$, $\forall t \neq t_{0}^{k}$. Therefore Lemma 2 holds if there exist some real constants that upper bound $\left\|\sigma_{\tilde{\theta}}\left(t_{0}^{k}\right)\right\|,\left\|\sigma_{\dot{\tilde{\theta}}}\left(t_{0}^{k}\right)\right\|, \forall k \in \mathbb{Z}$. The definition of $\theta_{d}(\cdot)$ (see (3)) allows us to write

$$
\begin{aligned}
\sigma_{\tilde{\theta}}\left(t_{0}^{k}\right) & =-\sigma_{\theta_{d}}\left(t_{0}^{k}\right)=-\sigma_{q_{d}}\left(t_{0}^{k}\right)-K^{-1} \sigma_{U_{r}}\left(t_{0}^{k}\right) \\
& =\left(\begin{array}{c}
\left(q_{d}\right)_{p}\left(t_{0}^{k-}\right) \\
0
\end{array}\right)-K^{-1} \sigma_{U_{r}}\left(t_{0}^{k}\right) \\
\sigma_{\dot{\tilde{\theta}}}\left(t_{0}^{k}\right) & =-\sigma_{\dot{\theta}_{d}}\left(t_{0}^{k}\right)=-\sigma_{\dot{q}_{d}}\left(t_{0}^{k}\right)-K^{-1} \sigma_{\dot{U}_{r}}\left(t_{0}^{k}\right) \\
& =\left(\begin{array}{c}
\left(\dot{q}_{d}\right)_{p}\left(t_{0}^{k-}\right) \\
0
\end{array}\right)-K^{-1} \sigma_{\dot{U}_{r}}\left(t_{0}^{k}\right) .
\end{aligned}
$$

Therefore

$$
\begin{aligned}
& \left\|\sigma_{\tilde{\theta}}\left(t_{0}^{k}\right)\right\| \leq\left\|\left(q_{d}\right)_{p}\left(t_{0}^{k-}\right)\right\|+\lambda_{\max }\left(K^{-1}\right)\left\|\sigma_{U_{r}}\left(t_{0}^{k}\right)\right\| \\
& \left\|\sigma_{\tilde{\tilde{\theta}}}\left(t_{0}^{k}\right)\right\| \leq\left\|\left(\dot{q}_{d}\right)_{p}\left(t_{0}^{k-}\right)\right\|+\lambda_{\max }\left(K^{-1}\right)\left\|\sigma_{\dot{U}_{r}}\left(t_{0}^{k}\right)\right\| .
\end{aligned}
$$

Using a (4) one obtains

$\sigma_{U_{r}}\left(t_{0}^{k}\right)=M(q) \sigma_{\ddot{q}_{r}}\left(t_{0}^{k}\right)+\sigma_{C(q, \dot{q}) \dot{q}_{r}}\left(t_{0}^{k}\right)-\gamma_{1} \sigma_{s_{1}}\left(t_{0}^{k}\right)$.

From (8) one has $\left(\dot{q}_{d}\right)_{n-p}(t)=0,\left(\ddot{q}_{d}\right)_{n-p}(t)=0 \forall t \in\left[\tau_{0}^{k}, t_{f}^{k}\right]$. Moreover, as we have mentioned at the end of Section 3, $\left(q_{d}\right)_{p}(\cdot)$, $\left(\dot{q}_{d}\right)_{p}(\cdot)$ and implicitly $\left(\ddot{q}_{d}\right)_{p}(\cdot)$ are set to zero on $\left(t_{0}^{k}, t_{f}^{k}\right]$. Thus 
taking into account that $\left\|\dot{q}\left(t_{0}^{k+}\right)\right\| \leq \mathbf{w}\left\|\dot{q}\left(t_{0}^{k-}\right)\right\|$ (where $\mathbf{w}=$ $\left.\sqrt{\frac{\lambda_{\max }(M)}{\lambda_{\min }(M)}}\right)$ one arrives at

$$
\begin{aligned}
\left\|\sigma_{\ddot{q}_{r}}\left(t_{0}^{k}\right)\right\| \leq & \left\|\left(\ddot{q}_{d}\right)_{p}\left(t_{0}^{k-}\right)\right\|+\gamma_{2}\left\|\left(\dot{q}_{d}\right)_{p}\left(t_{0}^{k-}\right)\right\| \\
& +\gamma_{2}(1+\mathbf{w})\left\|\dot{q}\left(t_{0}^{k-}\right)\right\| \\
\left\|\sigma_{C\left(q, \dot{q}^{\prime} \dot{q}_{r}\right.}\left(t_{0}^{k}\right)\right\| \leq & \left\|\sigma_{C(q, \dot{q})} \dot{q}_{r}\left(t_{0}^{k-}\right)\right\| \\
& +\left\|C\left(q, \dot{q}\left(t_{0}^{k+}\right)\right) \sigma_{\dot{q}_{r}}\left(t_{0}^{k}\right)\right\| \\
\in S(2(1+ & \left.\left.\gamma_{2}\right)\left\|\dot{q}\left(t_{0}^{k-}\right)\right\|\left\|\left(\dot{q}_{d}\right)_{p}\left(t_{0}^{k-}\right)\right\|+\gamma_{2}\left\|\left(q_{d}\right)_{p}\left(t_{0}^{k-}\right)\right\|\right) \\
\left\|\sigma_{s_{1}}\left(t_{0}^{k}\right)\right\| \leq & (1+\mathbf{w})\left\|\dot{q}\left(t_{0}^{k-}\right)\right\|+\left\|\left(\dot{q}_{d}\right)_{p}\left(t_{0}^{k-}\right)\right\| \\
& +\gamma_{2}\left\|\left(q_{d}\right)_{p}\left(t_{0}^{k-}\right)\right\| .
\end{aligned}
$$

When $V\left(\tau_{0}^{k}\right) \leq 1$, Lemma 1 states that $\left\|\left(\dot{q}_{d}\right)_{p}\left(t_{0}^{k-}\right)\right\|,\left\|\left(q_{d}\right)_{p}\left(t_{0}^{k-}\right)\right\|$ and $\left\|\dot{q}\left(t_{0}^{k-}\right)\right\|$ are bounded by some constants. Thus all the quantities in (A.2) are bounded by some constants independent of the cycle index $k$. This means that $\left\|\sigma_{U_{r}}\left(t_{0}^{k}\right)\right\|$ is bounded by a constant independent of the cycle index, which implies the same for $\left\|\sigma_{\tilde{\theta}}\left(t_{0}^{k}\right)\right\|$. In other words $\left\|\sigma_{\tilde{\theta}}(t)\right\| \in S(1)$. Differentiating (4) one obtains

$$
\begin{aligned}
\dot{U}_{r}(t)= & M(q) q_{r}^{(3)}(t)+\dot{M}(q) \ddot{q}_{r}(t)+C(q, \dot{q}) \ddot{q}_{r}(t) \\
& +\dot{C}(q, \dot{q}) \dot{q}_{r}(t)+\frac{\partial G}{\partial q} \dot{q}(t)-\gamma_{1} \dot{s}_{1}(t)
\end{aligned}
$$

where $\dot{M}, \dot{C}$ stand for $\frac{\mathrm{d} M}{\mathrm{~d} t}$ and $\frac{\mathrm{d} C}{\mathrm{~d} t}$ respectively.

It is clear that

$\dot{C}(q, \dot{q})(t)=\frac{\partial C}{\partial q}(q, \dot{q}) \dot{q}(t)+\frac{\partial C}{\partial \dot{q}}(q, \dot{q}) \ddot{q}(t)$

and using Properties 1 and 3 one derives

$\|\dot{C}(q, \dot{q})(t)\| \in S\left(\|\dot{q}(t)\|^{2}+\|\ddot{q}(t)\|\right)$.

Furthermore, Lemma 1 and the first equation in (1) assure that $\|\dot{q}(t)\|^{2},\|\ddot{q}(t)\| \in S(1)$. Thus $\|\dot{C}(q, \dot{q})(\cdot)\|,\left\|\sigma_{\dot{C}(q, \dot{q})}(\cdot)\right\| \in S(1)$ and one obtains

$$
\begin{aligned}
\left\|\sigma_{\dot{C}(q, \dot{q})}\left(t_{0}^{k}\right) \dot{q}_{r}\left(t_{0}^{k}\right)\right\| \leq & \left\|\sigma_{\dot{C}(q, \dot{q})}\left(t_{0}^{k}\right)\right\| \cdot\left\|\dot{q}_{r}\left(t_{0}^{k+}\right)\right\| \\
& +\left\|\dot{C}(q, \dot{q})\left(t_{0}^{k-}\right)\right\| \cdot\left\|\sigma_{\dot{q}_{r}}\left(t_{0}^{k}\right)\right\| \in S(1) .
\end{aligned}
$$

Property 1 allows us to replace $\dot{M}(q)$ by $C(q, \dot{q})+C^{\top}(q, \dot{q})$ which leads to

$$
\begin{aligned}
& \left\|\dot{M}(q) \ddot{q}_{r}(t)+C(q, \dot{q}) \ddot{q}_{r}(t)\right\| \leq 3\|C(q, \dot{q})\| .\left\|\ddot{q}_{r}(t)\right\| \\
& \quad \Rightarrow\left\|\dot{M}(q) \ddot{q}_{r}(t)+C(q, \dot{q}) \ddot{q}_{r}(t)\right\| \in S\left(\|\dot{q}\| \cdot\left\|\ddot{q}_{r}(t)\right\|\right) .
\end{aligned}
$$

Since $\left\|\ddot{q}_{r}(t)\right\| \leq\left\|\ddot{q}_{d}(t)\right\|+\gamma_{2}\|\dot{\tilde{q}}(t)\|$, using Lemma 1 one gets

$\left\|\dot{M}(q) \ddot{q}_{r}(t)+C(q, \dot{q}) \ddot{q}_{r}(t)\right\| \in S(1)$.

The definitions (6)-(8) and the first equation in (1) assure that $\left\|q_{r}^{(3)}(t)\right\| \in S(1)$. Therefore

$\left\|M(q) q_{r}^{(3)}(t)\right\| \leq \lambda_{\max }(M)\left\|q_{r}^{(3)}(t)\right\| \in S(1)$.

Property 2 states that $\left\|\frac{\partial G}{\partial q}\right\| \in S(1)$, which implies

$$
\left.\| \underset{\|\dot{q}(t)\| \in S(1)}{\left\|\frac{\partial G}{\partial q}(t)\right\| \in S(\|\dot{q}(t)\|)}\right\} \Rightarrow\left\|\frac{\partial G}{\partial q} \dot{q}(t)\right\| \in S(1) .
$$

Introducing (A.4)-(A.7) in (A.3) and taking into account the last inequality in (A.2) we arrive at $\left\|\sigma_{\dot{U}_{r}}(t)\right\| \in S(1)$ and thus $\left\|\sigma_{\dot{\tilde{\theta}}}(t)\right\| \in$ $S(1)$.

\section{Appendix B. Proof of Theorem 1}

First we observe that conditions (a) and (c) of Proposition 1 hold when the hypothesis of the Theorem is verified. Thus Theorem 1 holds if the conditions (b), (d) of Proposition 1 are verified.

(b) Using that $\dot{M}(q)-2 C(q, \dot{q})$ is a skew-symmetric matrix (see Property 1), straightforward computations show that on $\mathbb{R}_{+} \backslash$ $\bigcup_{k>0}\left[t_{0}^{k}, t_{f}^{k}\right]$ the time derivative of the Lyapunov function is given by

$$
\begin{aligned}
\dot{V}(t)= & -\gamma_{1}\|\dot{\tilde{q}}\|^{2}-\gamma_{1} \gamma_{2}^{2}\|\tilde{q}\|^{2}-\gamma_{1}\|\dot{\tilde{\theta}}\|^{2}-\gamma_{1} \gamma_{2}^{2}\|\tilde{\theta}\|^{2} \\
& -\gamma_{2}(\tilde{q}-\tilde{\theta})^{\top} K(\tilde{q}-\tilde{\theta})+\left(1+K_{f}\right) s_{1}^{\top} D_{p}^{\top}\left(\lambda-\lambda_{d}\right)_{p} \\
= & -\gamma_{1}\|\dot{\tilde{q}}\|^{2}-\gamma_{1} \gamma_{2}^{2}\|\tilde{q}\|^{2}-\gamma_{1}\|\dot{\tilde{\theta}}\|^{2}-\gamma_{1} \gamma_{2}^{2}\|\tilde{\theta}\|^{2} \\
& -\gamma_{2}(\tilde{q}-\tilde{\theta})^{\top} K(\tilde{q}-\tilde{\theta}) \leq 0
\end{aligned}
$$

where we have used the fact that $\left(q_{d}\right)_{p} \equiv 0,\left(\dot{q}_{d}\right)_{p} \equiv 0, q_{p} \equiv$ $0, \dot{q}_{p} \equiv 0$, thus $\left(s_{1}\right)_{p} \equiv 0$ on constraint phases and $\lambda_{p} \equiv 0$, $\left(\lambda_{d}\right)_{p} \equiv 0$ on free-motion phases. On the other hand

$$
\begin{aligned}
V(t) \leq & \frac{\lambda_{\max }(M(q))}{2}\left\|s_{1}\right\|^{2}+\frac{\lambda \max (J)}{2}\left\|s_{2}\right\|^{2}+\gamma_{1} \gamma_{2}\|\tilde{q}\|^{2} \\
& +\gamma_{1} \gamma_{2}\|\tilde{\theta}\|^{2}+\frac{1}{2}(\tilde{q}-\tilde{\theta})^{\top} K(\tilde{q}-\tilde{\theta}) \\
\leq & \gamma^{-1}\left[\gamma_{1}\|\dot{\tilde{q}}\|^{2}+\gamma_{1} \gamma_{2}^{2}\|\tilde{q}\|^{2}+\gamma_{1}\|\dot{\tilde{\theta}}\|^{2}+\gamma_{1} \gamma_{2}^{2}\|\tilde{\theta}\|^{2}\right. \\
& \left.+\gamma_{2}(\tilde{q}-\tilde{\theta})^{\top} K(\tilde{q}-\tilde{\theta})\right]
\end{aligned}
$$

where

$$
\begin{aligned}
& \gamma^{-1}=\max \left\{\frac{\lambda_{\max }(\mathbf{M}(q))\left(\gamma_{2}+2\right)+2 \gamma_{1}}{2 \gamma_{1} \gamma_{2}} ; \frac{1}{2 \gamma_{2}} ;\right. \\
& \left.\lambda_{\max }(\mathbf{M}(q)) \frac{1+2 \gamma_{2}}{2 \gamma_{1}}\right\}>0 \quad \text { with } \mathbf{M}(q)=\left(\begin{array}{cc}
M(q) & 0_{n \times n} \\
0_{n \times n} & J
\end{array}\right) .
\end{aligned}
$$

Therefore

$\dot{V}(t) \leq-\gamma^{-1} V(t)$ on $\mathbb{R}_{+} \backslash \bigcup_{k \geq 0}\left[t_{0}^{k}, t_{f}^{k}\right]$

(d) There is only one impact during each transition phase since $e=0$ and with the choice of $U_{t}^{B}$ in (4). Therefore $V\left(t_{\infty}^{k}\right)=V\left(t_{0}^{k-}\right)+$ $\sigma_{V}\left(t_{0}^{k}\right) \leq V\left(\tau_{0}^{k}\right)+\sigma_{V}\left(t_{0}^{k}\right)$. We compute now the jump of the Lyapunov function at the impact time $t_{0}^{k}$. Let $\mathcal{K}=\left(\begin{array}{cc}K & -K \\ -K & K\end{array}\right)$ and $\psi=\left(q^{\top}, \theta^{\top}\right)^{\top}$.

$$
\begin{aligned}
& V\left(t_{0}^{k+}\right)-V\left(t_{0}^{k-}\right)=\gamma_{1} \gamma_{2} \sigma_{\tilde{\psi}^{\top}} \tilde{\psi}^{(}\left(t_{0}^{k}\right) \\
& +\frac{1}{2}\left(s^{\top}\left(t_{0}^{k+}\right) \mathbf{M}(q) s\left(t_{0}^{k+}\right)-s^{\top}\left(t_{0}^{k-}\right) \mathbf{M}(q) s\left(t_{0}^{k-}\right)\right) \\
& \quad+\frac{1}{2}\left(\tilde{\psi}^{\top}\left(t_{0}^{k+}\right) \mathcal{K} \tilde{\psi}\left(t_{0}^{k+}\right)-\tilde{\psi}^{\top}\left(t_{0}^{k-}\right) \mathcal{K} \tilde{\psi}\left(t_{0}^{k-}\right)\right) .
\end{aligned}
$$

Replacing $\tilde{\psi}\left(t_{0}^{k+}\right)$ by $\tilde{\psi}\left(t_{0}^{k-}\right)+\sigma_{\tilde{\psi}}\left(t_{0}^{k}\right)$, the second term of the right hand side of (B.1) becomes

$\frac{1}{2}\left(2 \tilde{\psi}^{, \top}\left(t_{0}^{k-}\right) \mathcal{K} \sigma_{\tilde{\psi}}\left(t_{0}^{k}\right)+\sigma_{\tilde{\psi}}^{{ }^{\top}}\left(t_{0}^{k}\right) \mathcal{K} \sigma_{\tilde{\psi}}\left(t_{0}^{k}\right)\right)$

which is upper bounded by

$\lambda_{\max }(\mathcal{K})\left(\left\|\tilde{\psi}\left(t_{0}^{k-}\right)\right\| \cdot\left\|\sigma_{\tilde{\psi}}\left(t_{0}^{k}\right)\right\|+\frac{1}{2}\left\|\sigma_{\tilde{\psi}}\left(t_{0}^{k}\right)\right\|^{2}\right)$.

Therefore Lemmas 1 and 2 imply that there exists a real positive constant $c_{1}$ such that

$\frac{1}{2}\left(\tilde{\psi}^{, \top}\left(t_{0}^{k+}\right) \mathcal{K} \tilde{\psi}\left(t_{0}^{k+}\right)-\tilde{\psi}^{, \top}\left(t_{0}^{k-}\right) \mathcal{K} \tilde{\psi}\left(t_{0}^{k-}\right)\right) \leq c_{1}, \quad \forall k \geq 0$. 
On the other hand

$$
\begin{aligned}
& s^{\top}\left(t_{0}^{k+}\right) \mathbf{M}(q) s\left(t_{0}^{k+}\right)-s^{\top}\left(t_{0}^{k-}\right) \mathbf{M}(q) s\left(t_{0}^{k-}\right) \\
& \quad=\sigma_{s_{1}^{\top} M(q) s_{1}}\left(t_{0}^{k}\right)+\sigma_{s_{2}^{\top} J s_{2}}\left(t_{0}^{k}\right) .
\end{aligned}
$$

It is easy to see that

$\sigma_{s_{2}^{\top} J s_{2}}\left(t_{0}^{k}\right)=2 s_{2}^{\top}\left(t_{0}^{k-}\right) J \sigma_{s_{2}}\left(t_{0}^{k}\right)+\sigma_{s_{2}}^{\top}\left(t_{0}^{k}\right) J \sigma_{s_{2}}\left(t_{0}^{k}\right)$

and using Lemma 1, Lemma 2 and the relation $\sigma_{s_{2}}\left(t_{0}^{k}\right)=\sigma_{\dot{\tilde{\theta}}}\left(t_{0}^{k}\right)+$ $\gamma_{2} \sigma_{\tilde{\theta}}\left(t_{0}^{k}\right)$ one deduces that there exists a real positive constant $c_{2}$ such that

$\sigma_{s_{2}^{\top} J s_{2}}\left(t_{0}^{k}\right) \leq c_{2}, \quad \forall k \geq 0$.

As proved in Morărescu and Brogliato (2008) there exists a real positive constant $c_{3}$ such that

$\sigma_{s_{1}^{\top} M(q) s_{1}}\left(t_{0}^{k}\right)+\gamma_{1} \gamma_{2} \sigma_{\tilde{q}^{\top} \tilde{q}}\left(t_{0}^{k}\right) \leq c_{3}, \quad \forall k \geq 0$.

Finally, Lemma 2 assures the existence of $c_{4} \in \mathbb{R}_{+}$such that

$\gamma_{1} \gamma_{2} \sigma_{\tilde{\theta}^{\top} \tilde{\theta}}\left(t_{0}^{k}\right) \leq c_{4}, \quad \forall k \geq 0$

In conclusion, inserting (B.2)-(B.5) in (B.1) one gets

$V\left(t_{0}^{k+}\right)-V\left(t_{0}^{k-}\right) \leq c_{1}+c_{2}+c_{3}+c_{4}, \quad \forall k \geq 0$.

Thus condition (d) of Proposition 1 is verified for $\rho^{*}=1, \xi=$ $c_{1}+c_{2}+c_{3}+c_{4}$ and the closed-loop system (1), (3) and (4) is practically weakly stable with $R=\alpha^{-1}\left(\mathrm{e}^{-\gamma\left(t_{f}^{k}-t_{\infty}^{k}\right)}(1+\xi)\right)$.

Let us consider $\bar{\rho}=\min \left\{\lambda_{\min }(\mathbf{M}(q)) / 2 ; \gamma_{1} \gamma_{2}\right\}$. Defining $\alpha$ : $\mathbb{R}_{+} \mapsto \mathbb{R}_{+}, \alpha(\omega)=\bar{\rho} \omega^{2}$ we get $\alpha(0)=0, \alpha(\|[s(t), \tilde{q}(t)]\|) \leq$ $V(t, s, \tilde{q})$ and the proof is finished.

\section{References}

Acary, V., \& Brogliato, B. (2008). Lecture notes in applied and computational mechanics: Vol. 35. Numerical methods for nonsmooth dynamical systems. Berlin, Heidelberg: Springer-Verlag.

Bourgeot, J.-M., \& Brogliato, B. (2005). Tracking control of complementarity lagrangian systems. International Journal of Bifurcation and Chaos, 15(6), 1839-1866.

Brogliato, B. (1999). Nonsmooth mechanics (2nd ed.). London: Springer CCES.

Brogliato, B., Niculescu, S.-I., \& Orhant, P. (1997). On the control of finitedimensional mechanical systems with unilateral constraints. IEEE Transactions on Automatic Control, 42(2), 200-215.
Brogliato, B., Ortega, R., \& Lozano, R. (1995). Global tracking controllers for flexiblejoint manipulators: A comparative study. Automatica, 31(7), 941-956.

Dzonou, R., \& Monteiro Marques, M. D. P. (2007). A sweeping process approach to inelastic contact problems with general inertia operators. European Journal of Mechanics - A/Solids, 26(3), 474-490.

Galeani, S., Menini, L., Potini, A., \& Tornambè, A. (2008). Trajectory tracking for a particle in elliptical billiards. International Journal of Control, 81(2), 189-213.

Leine, R. I., \& van de Wouw, N. (2008a). Uniform convergence of monotone measure differential inclusions: With application to the control of mechanical systems with unilateral constraints. International Journal of Bifurcation and Chaos, 18(5), 1435-1457.

Leine, R. I., \& van de Wouw, N. (2008b). Lecture notes in applied and computational mechanics: Vol. 36. Stability and convergence of mechanical systems with unilateral constraints. Springer Verlag.

Mabrouk, M. (1998). A unified variational model for the dynamics of perfect unilateral constraints. European Journal of Mechanics A/Solids, 17(5), 819-842.

McClamroch, N., \& Wang, D. (1988). Feedback stabilization and tracking of constrained robots. IEEE Transactions on Automatic Control, 33, 419-426.

Morărescu, I.-C., \& Brogliato, B. (2008). Passivity-based tracking control of multiconstraint complementarity Lagrangian systems. In IEEE Conference on Decision and Control. See also http://hal.inria.fr/docs/00/27/62/64/PDF/RR6483.pdf.

Morărescu, I.-C., Brogliato, B, \& Hadd, S. (2008). Passivity-based switching control of flexible-joint complementarity mechanical systems. http://hal.inria.fr/docs/00/34/22/92/PDF/RR-6739.pdf. 